\title{
Miranda
}

Revue pluridisciplinaire du monde anglophone /

Multidisciplinary peer-reviewed journal on the English-

speaking world

$17 \mid 2018$

Paysages et héritages de David Bowie

\section{Introduction - New ways ever free}

Philippe Birgy, David Roche et Nathalie Vincent-Arnaud

\section{OpenEdition}

Journals

Édition électronique

URL : http://journals.openedition.org/miranda/12133

DOI : 10.4000/miranda.12133

ISSN : 2108-6559

Éditeur

Université Toulouse - Jean Jaurès

Référence électronique

Philippe Birgy, David Roche et Nathalie Vincent-Arnaud, «Introduction - New ways ever free », Miranda [En ligne], 17 | 2018, mis en ligne le 19 septembre 2018, consulté le 16 février 2021. URL : http://journals.openedition.org/miranda/12133; DOI : https://doi.org/10.4000/miranda.12133

Ce document a été généré automatiquement le 16 février 2021.

\section{$(1) \Theta$}

Miranda is licensed under a Creative Commons Attribution-NonCommercial-NoDerivatives 4.0 International License. 


\section{Introduction - New ways ever free}

\section{Philippe Birgy, David Roche et Nathalie Vincent-Arnaud}

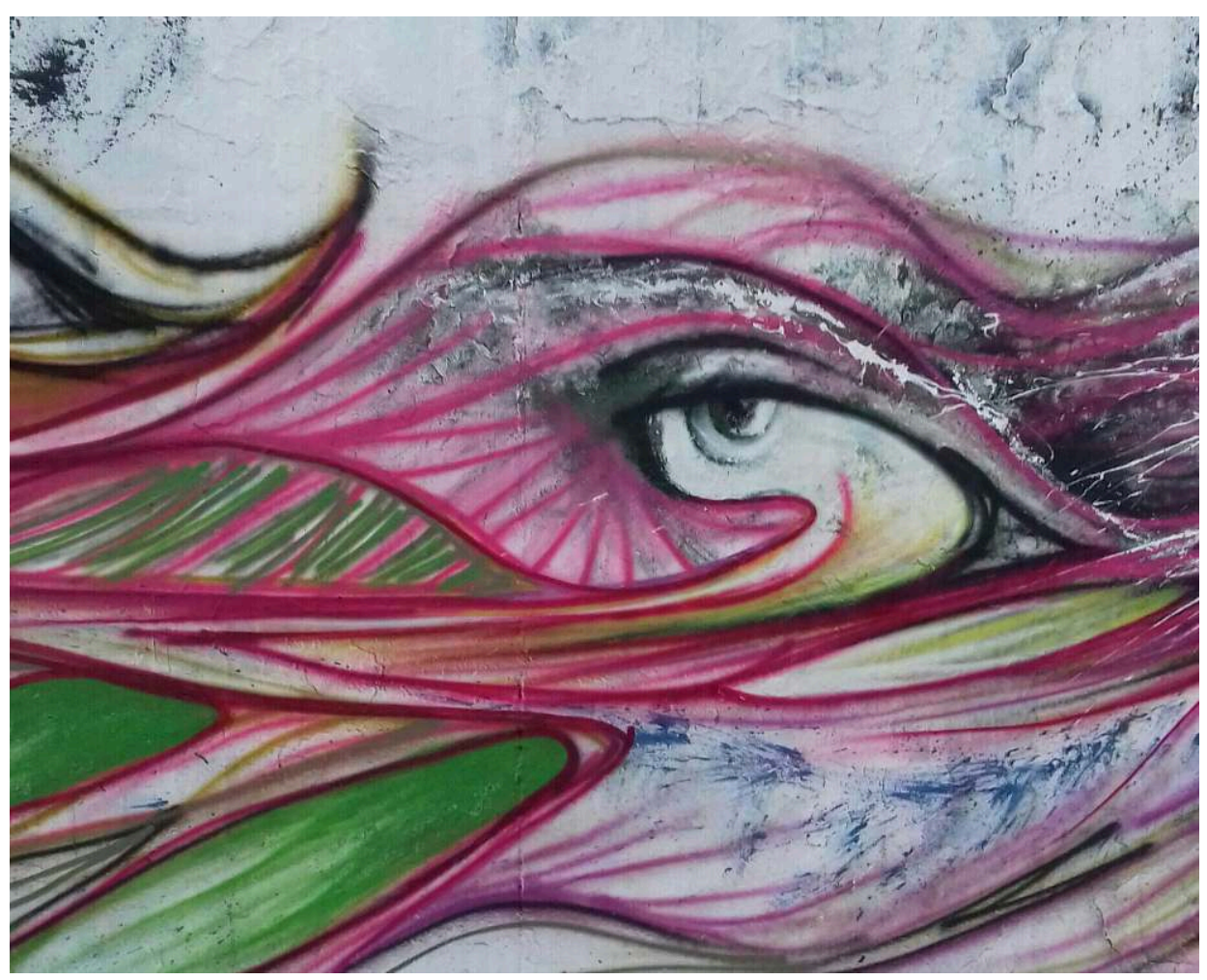

Street art à Mauerpark, Prenzlauer Berg, Berlin, août 2018

photo : N. Vincent-Arnaud

Ce numéro et la journée d'étude qui l'ont précédé sont évidemment nés d'un événement : la mort de David Jones, le 10 janvier 2016 à New York, suite à un cancer. Le choc ne résultait pas d'un acte brutal tel que ceux - assassinat, overdose, suicide - qui entrânèrent la disparition de tant de stars de l'histoire de la musique populaire, de Jimi Hendrix en 1970 à Chris Cornell en 2017, en passant par Janis Joplin (1970), Ian Curtis (1980), John Lennon (1980), Kurt Cobain (1994), Tupac Shakur (1996), Jeff Buckley (1997) et Biggie Smalls (1997). Sans doute la mort d'une star et/ou d'un artiste est-elle 
toujours un traumatisme, du fait notamment de la place fondatrice qu'elle occupe dans notre vie et du rôle qu'elle a joué et joue parfois encore dans notre construction de nous-mêmes. Mais peut-être la mort de Bowie se distinguait-elle par une singularité. Peut-être la sidération émanait-elle du fait que Bowie avait toujours été un être changeant, pluriel, en perpétuel renouveau et semblant de ce fait confiner à l'immortalité. De Ziggy (1972-1973) au Thin White Duke (1975-1976), du Bowie aux allures «straight » de Let's Dance (1983) au Bowie à l'apparence relativement lissée des années 1990-20001', l'artiste s'était construit comme l'« étoile noire» de la chanson éponyme de son dernier album sorti deux jours avant sa mort :

Something happened on the day he died

Spirit rose a metre and stepped aside

Somebody else took his place and bravely cried

"I'm a blackstar, I'm a blackstar."

2 Bowie ne meurt jamais mais renaît de ses cendres, tel le phénix. C'est le défi identitaire, à la fois esthétique et politique, lancé par Bowie, à la société et même à la mort, et le courage et l'obstination d'endurer qui est peut-être l'empreinte majeure de l'artiste. Au lendemain de sa disparition, les images et les voix n'ont cessé de se multiplier pour évoquer les paysages variés constitutifs de son œuvre, des styles musicaux aux personnages nés de son imagination en passant par les diverses facettes d'une carrière qui l'a vu, tour à tour ou simultanément, endosser plusieurs rôles, le menant de la musique au théâtre, au cinéma et aux arts plastiques, autant de domaines qu'il a cultivés avec passion et où il a su imprimer une marque indélébile et bien souvent décisive. De la poussière d'étoile à l'étoile noire en passant par toutes les formes de soleil noir où se déploient les personnages, l'œuvre de Bowie se fait traversée d'atmosphères, confluence de discours littéraires et artistiques, mémoire et anticipation de l'Histoire, exploration ininterrompue ouvrant des perspectives toujours plus nombreuses en matière d'art, de redéfinition du genre - quelle que soit l'acception du terme - et de discours sur l'humain. La plaque commémorative officielle apposée, dès août 2016, sur la façade de son ancien domicile berlinois, 155 Hauptstrasse, et mentionnant le célèbre refrain de « Heroes » (« We can be heroes, just for one day $»^{2}$ ) porte témoignage de ce qui est désormais appréhendé comme la valeur performative de l'œuvre de Bowie, œuvre explorant sans relâche les confins des ruines et des rêves, des désarrois et des désirs, pour faire surgir l'humain à un nouveau jour ; objet de tant de mises en scène depuis le maquillage outrancier de "Life on Mars?" (1971) jusqu'au regard surnaturel de Heathen (2002) en passant par l'éclair et le cercle sur le front d'Alladin Sane (1973), son œil ne semble-t-il pas encore et toujours à l'œuvre dans nombre de paysages toujours renouvelés de street art qui défilent sur les murs de Prenzlauer Berg, Kreutzberg ou de l'East Side Gallery ? Autant de points disséminés qui, d'un continent à un autre, d'un langage, art ou média à un autre, ne cessent de tisser l'étoffe moirée d'un héritage dont le présent numéro, en dépit de ses perspectives variées, ne prétend aucunement épuiser l'inventaire.

3 Notre journée d'étude du 2 décembre 2016 a offert deux expressions personnelles de ce que Bowie pouvait représenter pour chacun: en point d'orgue de la journée, un spectacle mêlant danse, musique, théâtre et poésie, intitulé " New Ways Forever Free », conçu par Nathalie Vincent-Arnaud ${ }^{3}$, et, auparavant, une communication ethnoautobiographique présentée par Georges-Claude Guilbert, spécialiste de celebrity studies et de gender studies. Guilbert a expliqué comment Bowie l'avait accompagné pendant différentes étapes de sa vie: du jeune queer épris de science-fiction et versant dans 
certains excès dans le Manchester des années 1970, à celui qui quelques années plus tard trouva la force de mettre fin auxdits excès, puis au professeur agrégé qui décida de se lancer dans une thèse, et enfin à l'enseignant-chercheur qui s'est livré devant nous ce jour-là. Ce faisant, Guilbert nous a ainsi rappelé l'intime corrélation entre la trajectoire d'une recherche et le parcours intime et sensible d'une subjectivité humaine, et plus généralement, comme son mentor, entre la construction d'une œuvre et la construction de soi. La sincérité de la communication de Guilbert a amené Emeline Jouve, co-organisatrice de la journée, à demander aux étudiants dans le public en quoi Bowie, star des années 1970 et 1980, était toujours autant d'actualité pour eux. Plusieurs ont réagi, et Chloé Monasterolo, doctorante à l'Université Toulouse-Jean Jaurès, a alors proposé l'hypothèse suivante: peut-être n'était-ce n'est pas tant le musicien qui leur plaisait que l'idée de David Bowie elle-même. Cette hypothèse tout aussi séduisante que convaincante rejoint les propos de Gary Kemp qui, à propos de l'exposition « David Bowie Is » de 2013, affirmait : «It's not about one man ; it's about all of us. $\aleph^{4}$ Le caractère elliptique de la phrase-titre de cet événement qui a fait date a été largement commenté par la presse à l'époque, l'absence d'attribut ouvrant sur des possibilités d'instanciation infinies que même le contenu foisonnant de l'exposition, son agencement en salles, recoins, reliques multiples n'ont pu épuiser ; pas plus que n'a pu le faire l'exposition-vente «Life on Mars » qui, par un hasard de calendrier peu commun, s'est tenue au Multiple (le bien nommé) de Toulouse à partir du 2 décembre 2016, y présentant 40 photographies inédites de Mick Rock, photographe légendaire de l'artiste, ainsi que divers objets retraçant certaines étapes de sa carrière. ${ }^{5}$ Mais chercher désespérément à dresser une liste d'attributs pour combler ce que l'on pense être une vacance syntaxique du titre est peut-être une entreprise aussi erronée qu'illusoire, la simple non-instanciation, la simple prédication d'existence ne faisant après tout que dire l'essentiel : David Bowie existe. Il existe à travers les innombrables images et constructions mentales qui sont devenues son héritage. Et c'est cette existence qui, au grand jour ou de manière subreptice, est venue imprimer aux paysages à travers lesquels défilent nos vies, sur lesquels elles s'inscrivent, un relief qui sait, de manière renouvelée, faire l'amalgame de l'étrange et du familier, accueillir évolutions et mutations : le refrain de "Changes » (1971), «Ch-ch-ch-ch-changes / Turn and face the strange ", résonne comme une invitation renouvelée à cette odyssée existentielle.

4 Corrélativement, la singularité de chaque parcours humain peut trouver dans le paysage déroulé par le nom de Bowie matière à s'éclairer, se nourrir, s'animer, se renouveler, se comprendre, s'accepter et, à son tour, faire œuvre à travers de multiples et toujours uniques "phrasés du vivre » (Macé 2016). Le domaine littéraire n'est pas en reste, et aux multiples histoires créées, réécrites, ré-ensemencées par Bowie succèdent les histoires qu'il a inspirées, dont il est, en filigrane, le héros. Au nombre de celles-ci figure, en France, le roman de Sonia David au titre détonant, David Bowie n'est pas mort (2017), dans lequel la mort de Bowie, en contrepoint de la disparition des deux parents de l'héroïne, déclenche tout un périple mémoriel dont la phrase «Mickey Mouse has grown up a cow » («Life on Mars?»), est le viatique, ouvrant plus largement encore l'horizon d'une relation entre sœurs partiellement inexplorée entre abandons, pertes, désirs refoulés, métamorphoses. D'autres aspects du questionnement identitaire sont à l'honneur dans De l'influence de David Bowie sur la destinée des jeunes filles, roman de JeanMichel Guenassia qui a également vu le jour en 2017, explorant à la loupe le parcours 
d'un adolescent en proie aux tiraillements du genre et aux éblouissements qu'ils font naître.

5 Artiste polyvalent, "caméléon musical " ${ }^{6}$, icône, star, ensemble de personae, palimpseste, parangon de la célébrité, de la performance, du postmodernisme, du genre comme construction ${ }^{7}$, David Bowie est un objet d'études de choix pour de nombreuses disciplines en sciences humaines. Si quelques travaux universitaires lui avaient déjà été consacrés (Waldrep 2004, Stevenson 2006), la période 2012-2013 marque la naissance des Bowie studies. Le premier colloque international, "Strange Fascination? A Symposium on David Bowie ", a lieu en octobre 2012, à l'Université de Limerick en Irlande. En mars 2013 se tient l'exposition "David Bowie Is " précédemment mentionnée, au Victoria and Albert Museum à Londres ${ }^{8}$, première étape d'un itinéraire de cinq années qui la mènera, station to station, du Martin-Gropius-Bau à Berlin à la Philarmonie de Paris, à Chicago, Tokyo, Melbourne, jusqu'au Brooklyn Museum en 2018. Mars 2013 est aussi le mois de la sortie de The Next Day, premier album de Bowie depuis Reality en 2003... et des inquiétudes concernant sa santé. Les publications se sont multipliées depuis : David Bowie: Critical Perspectives (2015), dirigé par Eoin Devereux, Aileen Dillane et Martin J. Power, qui fait suite au colloque de 2012 ; Future Nostalgia : Performing David Bowie (2015) de Shelton Waldrep ; Forever Stardust: David Bowie Across the Universe (2017) de Will Brooker; ainsi que l'«In Focus : David Bowie On-Screen » paru dans Cinema Journal (2018). Enfin, le 22 mars 2016, s'est tenue à l'Université d'EvryVal d'Essonne une demi-journée d'étude en hommage à David Bowie, organisée par le musicologue Grégoire Tosser, avec la participation de Matthieu Thibault, auteur de David Bowie : l'avant-garde pop (2013). Ce numéro de Miranda et la journée d'étude dont il émane se sont à l'évidence inscrits dans cette dynamique. Ils ont adopté une perspective résolument pluridisciplinaire (études en audiovisuel, histoire culturelle, musicologie, philosophie, réception, star studies), reflet des multiples facettes et talents d'un artiste tout à la fois "mâle au féminin ", " un peu Dorian Gray » et « légèrement fêlé ", selon un texte de Serge Gainsbourg9 qui, le temps d'une chanson (certes largement éclipsée depuis), l'a lui aussi célébré à sa manière.

6 L'appel de l'étrange résonne dans ses œuvres jusqu'à être la signature de nombre d'albums, de performances, de clips, de morceaux emblématiques, ses manifestations principales étant les innombrables figures d'ouverture qui constellent l'ensemble. Aux thèmes de l'essor et de l'échappée se conjuguent ceux de la fêlure, de l'errance et de l'aliénation, auxquels la musique elle-même fait écho de manière constante par la multiplicité de ses influences, de ses redécoupages stylistiques, du surgissement de l'inédit via notamment la plasticité d'une voix qui emprunte elle aussi tous les carrefours de l'étrangeté, du transgénérique, du transfrontalier : des aigus déchirants de « Ziggy Stardust» (1972) au falsetto de "Ashes to Ashes » (1980) sombrant l'instant d'après dans le registre le plus grave en un jeu de figuralisme spectaculaire (« Hitting an all-time low»), de la voix dédoublée de "The Bewlay Brothers» (1971) aux inflexions de crooner de "Let's Dance» (1983), du murmure au cri, elle ne fait que révéler son ambitus et ses innombrables potentialités d'adaptation à une thématique, à une ambiance, à la nécessité de l'œuvre qu'elle sert - on songe ici inévitablement à la somptueuse prestation théâtrale de Bowie dans l'adaptation par John Pomerance d'Elephant Man en 1977. Aux détours de cette voix répondent ceux d'une création musicale qui n'a fait que voyager, mêler héritages et trouvailles, du proto-punk de "Queen Bitch» (1971) à la soul de "Young Americans" (1975) en passant par les inflexions jazz et blues de "Cracked Actor » (1973) et de "Alladin Sane », les effluves 
psychédéliques de « Memory of a Free Festival » (1969), la spirale en forme de mantra de «Cygnet Committee» (1969), le lyrisme de "Lady Grinning Soul» (1973), les syncopes cuivrées de «Let's Dance » (1973) et les sonorités cold wave de l'album Low, fruit des années berlinoises de Bowie et de sa collaboration fructueuse avec Brian Eno. Une œuvre ouverte à plus d'un titre, explorant le passé et inspirant l'avenir comme en attestent les innombrables reprises de certains morceaux qui se sont peu à peu érigés en emblèmes tels que "Heroes", dont Coldplay et Gang of Youths ont proposé leurs propres interprétations, ou «Life on Mars", dont une des lectures les plus récentes, a cappella, a été offerte, en 2017, par l'ensemble vocal français Perspectives dans leur disque Songs of Experience ${ }^{10}$. Musicien polyvalent (chant, claviers, guitare, saxophone, etc.), résolument plasticien dans son approche, Bowie a modelé une œuvre dont sa persona fait partie comme un tout non fini, comme un processus en cours, comme une expérience ouverte tant aux contraintes génériques (cold wave, électro, funk, pop, rock psychédélique, soul, etc.) qu'aux tendances actuelles et aux contingences du cut-up burroughsien ${ }^{11}$, produisant ainsi un ensemble dont la variété est telle que chacun a "son » Bowie préféré... et qu'il est même possible d'en adorer un et d'en détester un autre tout en aimant quand même David Bowie.

7 La présence de Bowie à travers le domaine audiovisuel (cinéma, télévision) est d'une richesse incomparable. Comme d'autres artistes, bien sûr, il est une présence à la fois musicale (ses chansons figurent sur de nombreuses bandes sons) et culturelle (lors de la journée d'études, Matthew Leggett avait abordé la série Life on Mars [BBC, 2006-2007 ; $A B C, 2008-2009]$, mélange de polar et de science-fiction qui se déroule en 1973). Mais dans le cas de Bowie, il s'agit bel et bien d'une présence délibérée, tant par sa carrière d'acteur que par son travail visuel et audiovisuel, liée à sa volonté de "devenir un médium $»^{12}$. Cette volonté émane à n'en pas douter, au moins partiellement, de ses expériences successives de mime et de peintre: celles-ci n'ont cessé d'irriguer l'ensemble de ses pratiques artistiques, tout comme son attrait pour les chansons de Kurt Weill - dont il reprit magistralement Moon of Alabama en 1978 - trouve son origine dans sa fascination pour un expressionnisme pictural et cinématographique qui s'incarne massivement dans ses pochettes de disques et ses personae, de Station to Station à Heroes, du cracked actor au Thin White Duke et à son avatar mélancolique de Low.

Il semble ainsi tout naturel que Bowie occupe une place centrale dans l'histoire d'une forme encore naissante à la fin des années soixante : le clip vidéo. Dès 1969, le court métrage promotionnel Love You Till Tuesday, réalisé par Malcolm J. Thompson, propose un premier clip de "Space Oddity »" influencé à la fois par l'esthétique psychédélique (look hippie, effets optiques, filtres colorés) et par les décors d'un film qui a profondément marqué Bowie, 2001 : A Space Odyssey (Stanley Kubrick, 1968). On retrouve d'ailleurs l'imaginaire fantastique et $\mathrm{SF}$ ainsi que l'esthétique psychédélique jusque dans le clip de «Blackstar $»^{14}$ (Johan Renck, 2016). Le clip de «Space Oddity » de $1972^{15}$, réalisé cette fois-ci par le photographe Mick Rock, relève d'un autre genre: le clip tourné en studio, avec un Bowie chantant face à la caméra avec une guitare folk, la table de mixage renvoyant à la technologie décrite dans les paroles. La deuxième version participe donc largement à la réinvention à la fois du morceau (l'orchestration plus ample, la voix plus grave) et de Bowie lui-même (ici en Ziggy Stardust). Ce retour sur Bowie est un fait récurrent dans les clips plus récents, "Love Is Lost »16 (David Bowie, Jimmy King et Corinne Schwab, 2013) revenant, par exemple, sur la figure de Pierrot présente dans le célèbre clip de "Ashes to Ashes ${ }^{17}$ (David Mallet, 1980) qui évoquait quant à lui le Major Tom de "Space Oddity». Chaque clip est donc une 
ramification du travail de performance de Bowie, au même titre que les couvertures et les concerts, avec un souci du costume, de la coiffure, du maquillage ou du masque ${ }^{18}$ les clips de "Life on Mars » ${ }^{19}$ (Mick Rock, 1973) et de " "Heroes" " ${ }^{20}$ (Stanley Dorfman, 1977) figurent Bowie chantant seul dans un décor épuré (arrière-plan blanc et contrejour). Chaque clip est aussi un prolongement du morceau lui-même. Dans son étude du clip de «China Girl $»^{21}$ (David Mallet, 1983), Shelton Waldrep affirme que la dimension orientaliste, et donc idéologiquement problématique, de la reprise de Bowie, appropriation d'une chanson qui revêtait pour Iggy Pop un caractère plus personnel, ne peut être rachetée qu'en faisant appel au reste de la production artistique de Bowie de 1983 : soit à un autre clip, "Let's Dance »22 (David Mallet, 1983), où l'évocation de la situation sociale des aborigènes d'Australie y prend à contrepied la légèreté toute apparente du tube (la danse est ainsi entendue au sens métaphorique d'une « rencontre entre deux cultures » Vernallis 147) ; soit à la déconstruction de l'orientalisme dans le film Merry Christmas, Mr. Lawrence de Nagisa Ôshima ${ }^{23}$. Les articles de Jonathan Broda et de Thomas Schmitt sur les apparitions audiovisuelles de Bowie de 1969 à 1983, et celui de Robin Cauche sur "Where Are We Now $»^{24}$ (Tony Oursler, 2013) qui figurent dans ce numéro prolongent ainsi cette réflexion sur l'apport de Bowie à la forme du clip, sur Bowie comme « vidéo star » et sur le rôle du clip dans la construction de Bowie.

9 Si la filmographie de Bowie est somme toute assez mineure pour un acteur, elle est remarquable pour une star du rock, comme le remarque Mehdi Derfoufi ${ }^{25}$, suffisamment importante en tout cas pour que la revue Cinema Journal y consacre un dossier spécial et que trois articles de l'ouvrage David Bowie: Critical Perspectives s'y intéressent. Avec deux films majeurs, The Man Who Fell to Earth (Nicolas Roeg, 1976) et Merry Christmas, Mr. Lawrence, la carrière d'acteur de Bowie est largement plus conséquente que celle de la majorité des stars qui ont tenté l'aventure devant la caméra, qu'il s'agisse de Mick Jagger dans Performance (Donald Campbell et Nicolas Roeg, 1970), de Michael Jackson dans Moonwalker (Jerry Kramer et Colin Chilvers, 1988), d'Eminem dans 8 Mile (Curtis Hanson, 2002), ou bien encore de Michael Jordan dans Space Jam (Joe Pytka, 1996). Seul le parcours de Madonna, de Desperately Seeking Susan (Susan Seidelman, 1985) à Swept Away (Guy Ritchie, 2002), peut rivaliser avec celui de Bowie. En plus du talent, ceci s'explique certainement par le fait que Madonna comme Bowie ne cultivait pas une seule et même image. ${ }^{26}$

La dissémination cinématographique de Bowie est elle aussi un prolongement de son travail sur ses multiples personae, mais elle est rendue possible par sa propre formation de comédien et surtout de $\mathrm{mime}^{27}$ - c'est d'ailleurs ce talent-là qu'exploitent à plusieurs reprises Merry Christmas, Mr. Lawrence (Figure 1) et le numéro musical de Bowie dans Absolute Beginners (Julian Temple, 1986). Tous les films jouent sur l'attente de l'apparition de la star dans un rôle principal ou secondaire, que celle-ci survienne au début du film comme dans The Man Who Fell to Earth, The Hunger (Tony Scott, 1983) et Mr. Rice's Secret (Nicholas Kendall, 1999), qu'elle introduise un nouvel acte comme dans Merry Christmas, Mr. Lawrence, Labyrinth (Jim Henson, 1986) et Basquiat (Julian Schnabel, 1996), ou bien même qu'elle intervienne au milieu du film comme dans Absolute Beginners, voire dans l'épilogue comme dans Bandslam (Todd Graff, 2009). L'entrée de Thomas Jerome Newton, du Major Jack Celliers, de John Blaylock, de Jareth, d'Andy Warhol, de Vendice Partners, l'entrée de tous les personnages interprétés par Bowie est toujours exceptionnelle. Et elle se fait le plus souvent sur un mode théâtral : Bowie franchit les portes du tribunal dans Merry Christmas, Mr. Lawrence, sort d'une voiture dans Basquiat, d'un ascenseur dans Twin Peaks: Fire Walk With Me (David Lynch, 1992), 
d'une déchirure dans le réel dans Twin Peaks : Fire Walk With Me et Labyrinth (Figure 2). A chaque fois, une dramaturgie spécifique est mise en place pour marquer le moment où l'on voit enfin la star. Cette dramaturgie est centrée sur son visage, visage connu de tout le public, qu'il soit fan de Bowie ou non, à travers les pochettes de Hunky Dory, Alladin Sane et "Heroes" et les couvertures de magazines comme le célèbre « David Bowie Straight » de Rolling Stone du 12 mai 1983. Il s'agit plus précisément d'une dramaturgie du dévoilement. L'apparition de Newton dans The Man Who Fell to Earth donne le la: le personnage, figure sombre aux traits invisibles, déambule le long d'une pente, puis est aperçu avançant de dos au bord d'une route, cagoulé, avant que son visage ne soit révélé (Figure 3). Dans The Hunger, le montage, qui alterne entre gros plans des visages des artistes et du public, nous invite à repérer les stars (Bowie mais aussi Catherine Deneuve) lors d'un concert de Bauhaus, tâche compliquée à la fois par l'éclairage stroboscopique mais aussi par le fait que Bowie pourrait se trouver aussi bien dans le public que sur scène. La séquence d'ouverture annonce ainsi la centralité du visage comme motif et thème: John le vampire, qui pense avoir déjà vieilli de 30 ans en 24 heures, continue à vieillir à vue d'œil dans la salle d'attente de Sarah Roberts (Figure 4.1-4.2). Baal (Alan Clarke, 1982) procède à une défiguration similaire de la star, métamorphosée en artiste bohémien crasseux (Figure 7).

Fig. 1

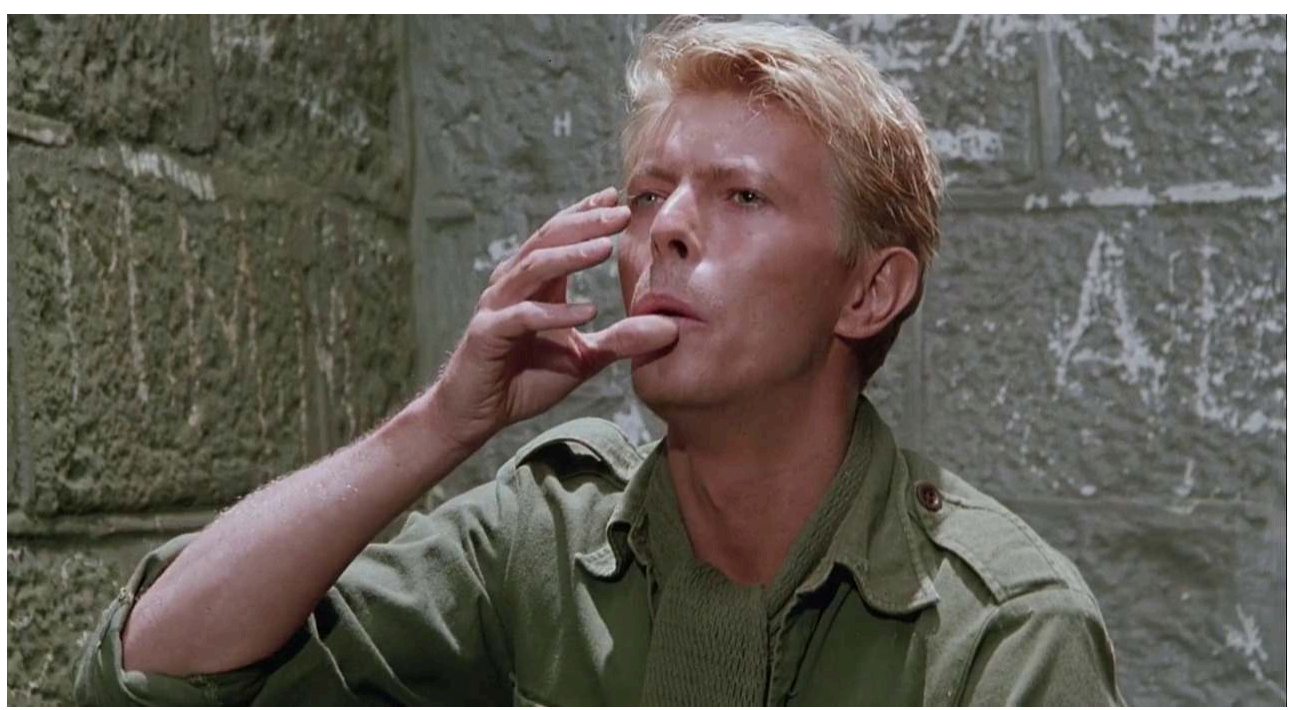

Dans sa cellule, le Major Celliers maintient les rituels du lever à travers le jeu dans Merry Christmas, Mr. Lawrence (Nagisa Ôshima, 1983). 
Fig. 2

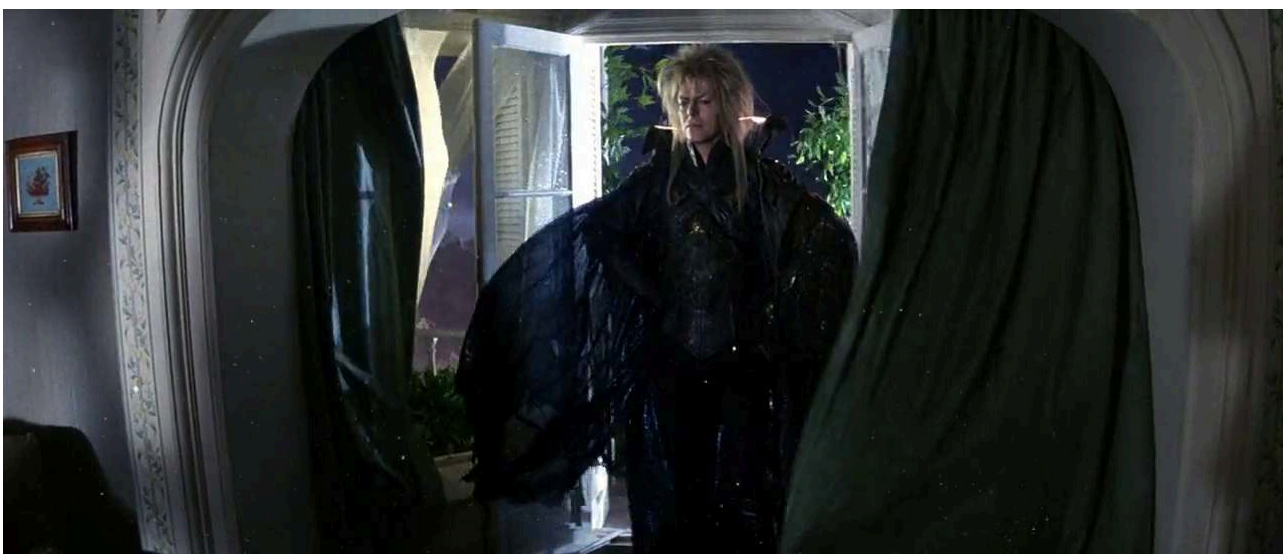

L'apparition théâtrale de Jareth dans Labyrinth (Jim Henson, 1986).

Fig. 3

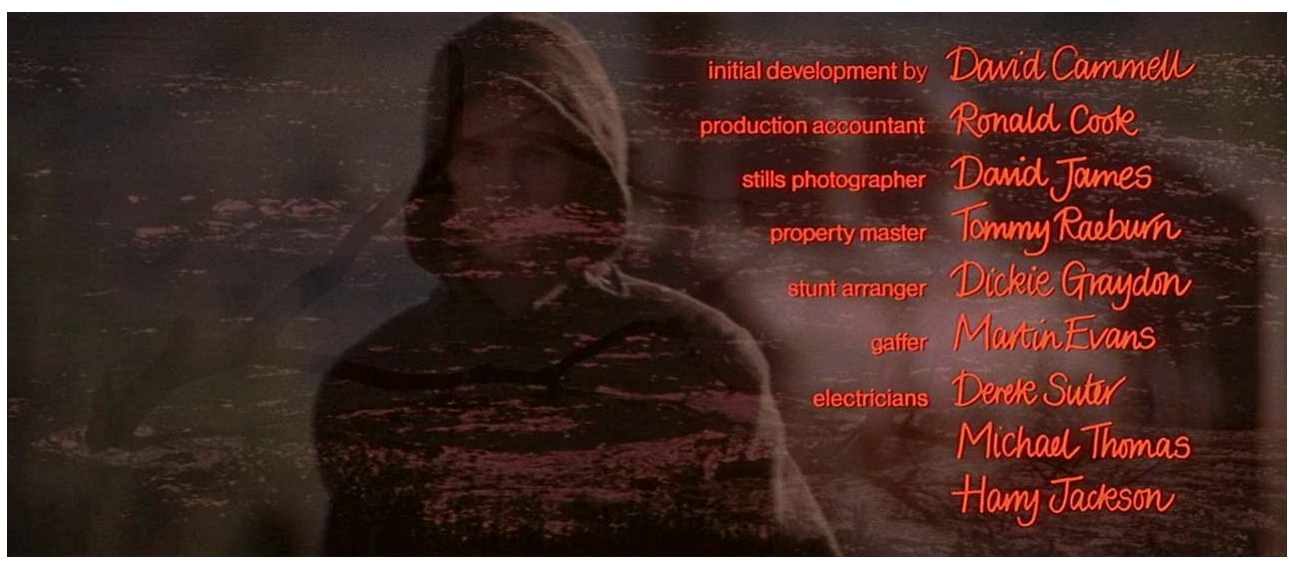

L'apparition voilée de Thomas Jerome Newton, l'extra-terrestre de The Man Who Fell to Earth (Nicolas Roeg, 1976).

Fig. 3.1-3.2

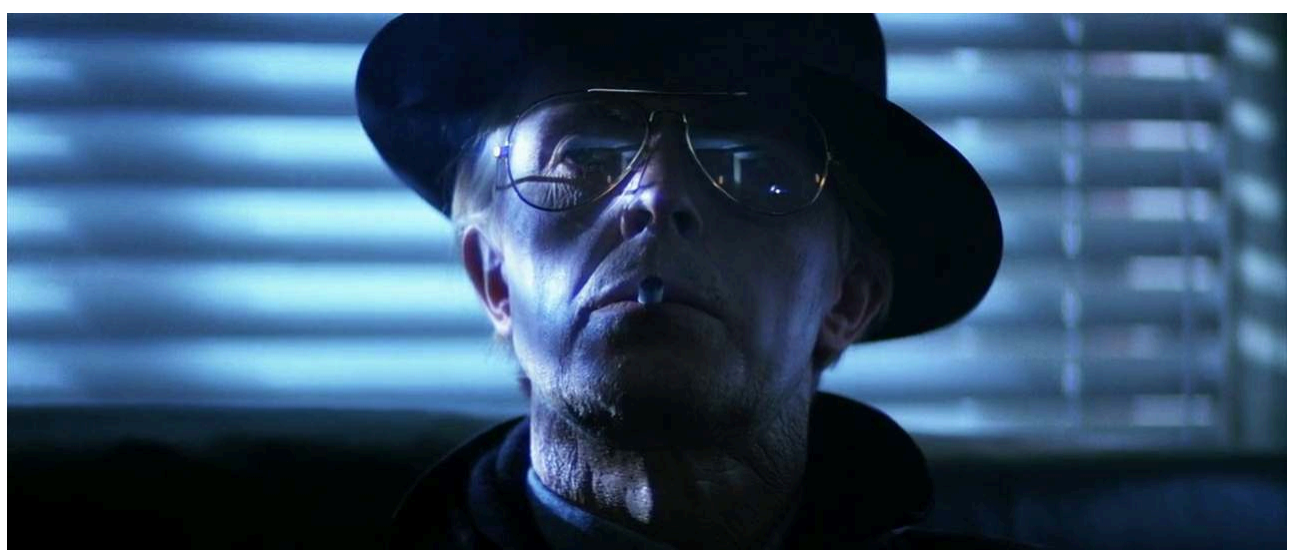




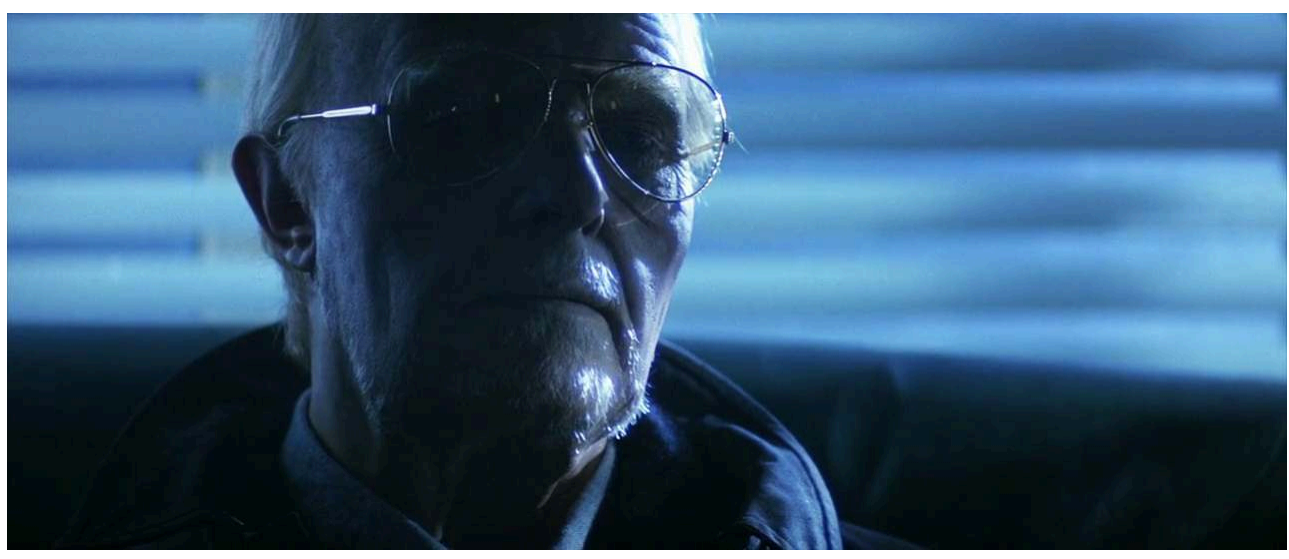

Bowie vieillit à grande vitesse dans The Hunger (Tony Scott, 1983).

11 Cette dramaturgie du dévoilement participe donc d'un suspense à deux inconnues: l'apparition de Bowie est certaine, mais l'on se demande quand elle va se produire et surtout quel David Bowie va faire son apparition. C'est en cela que cette dramaturgie est proprement jubilatoire. Tous les films problématisent l'image de Bowie. Avec ses cheveux rouges, Newton fait évidemment appel à la figure d'un autre extra-terrestre, Ziggy Stardust, avec des allures de Thin White Duke (Figure 4). Paul Ambrosius von Przygodski, antihéros du pénible Just a Gigolo (David Hemmings, 1978), évoque le sinistre Thin White Duke et son attirance pour le fascisme. John Blaylock et Major Celliers jouent de la nouvelle image emblématique des années 1980 du David Bowie, blond, séduisant, hétérosexuel, éminemment britannique, tandis que Vendice Partners incarne un système qui incite les artistes (comme Colin et Suzette) à se vendre corps et âme (comme Ambrosius von Przygodski); les nombreuses brèves apparitions (" cameos ») de Bowie dans des films des années 1990 renvoient souvent au Bowie de Let's Dance, dans une perspective critique en ce qui concerne Zoolander (Ben Stiller 2001), par exemple, puisque Bowie y apparaît comme le grand prêtre du faux (Redmond 154). Ou bien c'est avec une pointe d'ironie qu'il est convoqué, comme dans Twin Peaks: Fire Walk With Me (Redmond 152), sa chemise hawaïenne suggérant que l'agent Philip Jeffries n'était peut-être pas perdu dans une autre dimension mais seulement parti en vacances prolongées ! La relation entre Bowie et Warhol est à la fois biographique - les deux hommes se sont bel et bien rencontrés - et esthétique, Bowie ayant été influencé par le Pop Art et ayant même composé une chanson en hommage à l'artiste américain en 1971. Dans les films, la (con)fusion entre Bowie et son image est fréquemment mise en abyme à travers l'emploi de miroirs, comme dans The Man Who Fell to Earth, Absolute Beginners (Figure 5) et surtout dans Labyrinth où la confrontation finale entre l'héroïne et Jareth se déroule dans une sorte de palais des glaces sans glaces. Elle suggère que la star est bel et bien ce que Gilles Deleuze appelle une "image-cristal », dont l'image actuelle se confond avec son (ou ses) image(s) virtuel(les) (Deleuze 92-95). 
Fig. 4

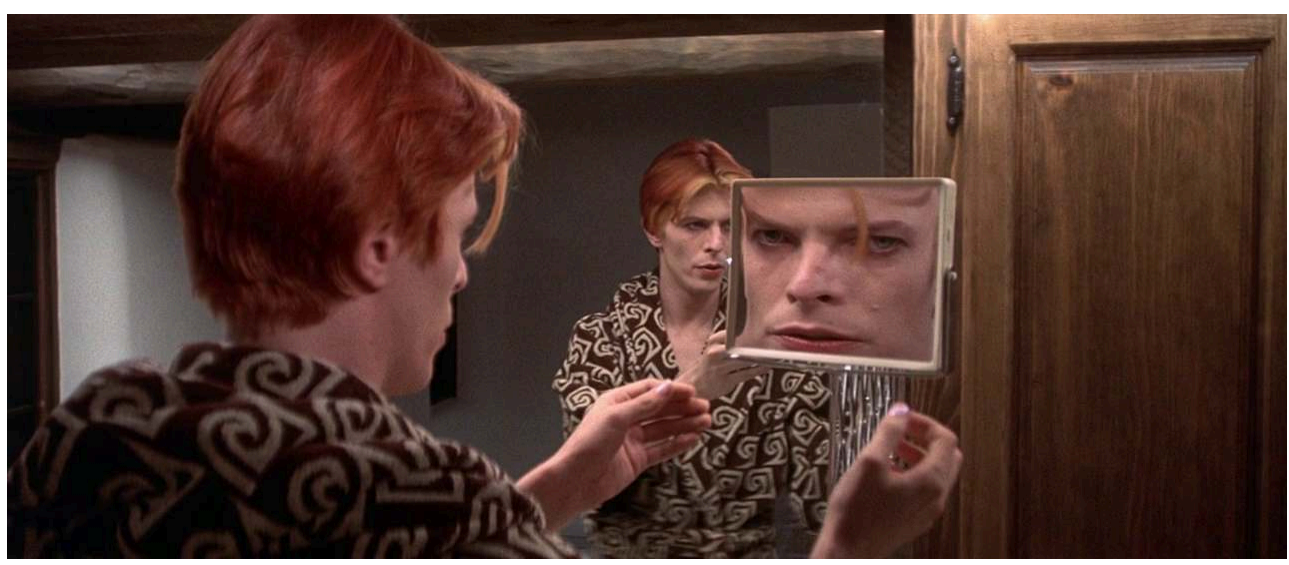

Newton, l'extra-terrestre, regarde son reflet qui regarde le spectateur le regarder dans The Man Who Fell to Earth.

Fig. 5

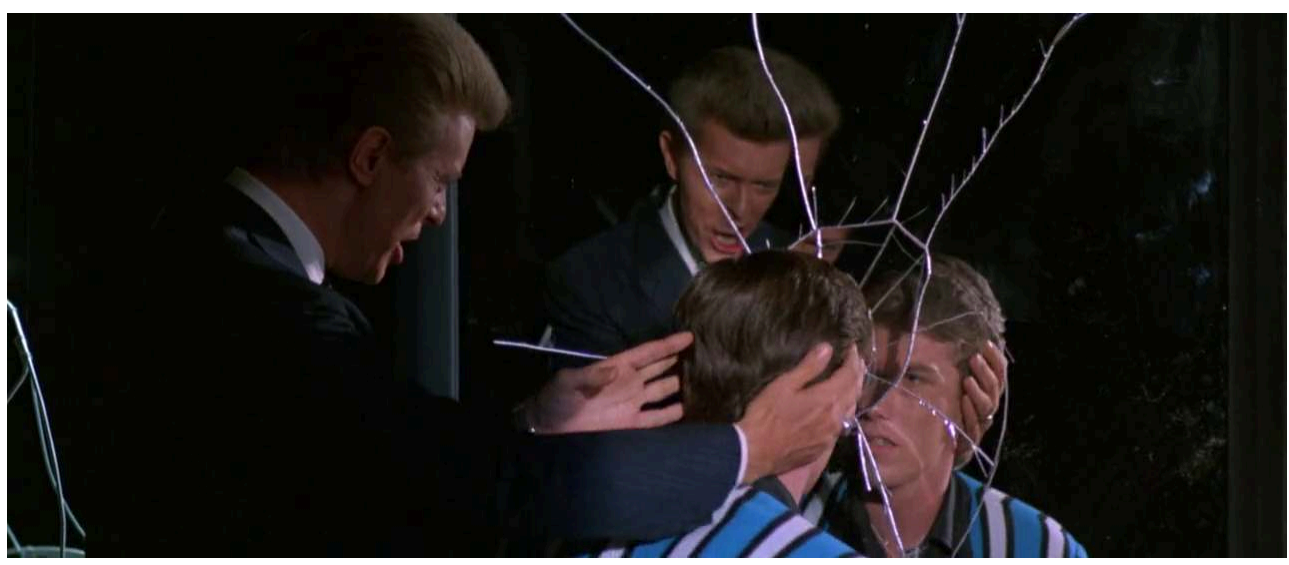

Vendice Partners incite le protagoniste Colin à devenir un vendu du système dans Absolute Beginners (Julian Temple, 1986).

12 Les films puisent dans les différentes facettes de la «star image $»^{28}$ de Bowie, celle-ci étant constituée de l'ensemble des occurrences médiatiques (clips, concerts, entretiens, films, publicité, etc.) de la star. Il incarne les figures de la magie (Jareth, Tesla dans The Prestige [Christopher Nolan, 2006]) comme celles de l'artiste (Baal, Warhol), sa polyvalence le rendant crédible dans la peau d'un poète prolétaire autant que dans celle d'un guru du Pop Art. Les films des années 1970 et 1980 puisent largement dans l'ambivalence sexuelle associée à Bowie, comme Julie Lobalzo Wright et Rosalind Galt l'ont montré29 ${ }^{\prime}$ l'humanisation de Newton, extra-terrestre dépourvu d'organes sexuels, passe par une allégeance aux normes hétérosexuelles, selon Wright ; John Blaylock est peut-être hétérosexuel, mais sa compagne Miriam le remplace par une femme ; Jareth incarne aussi bien la star masculine désirée par une adolescente que le pédophile qui la poursuit (Galt 137) ; Celliers est en quelque sorte la version queer du Colonel Nicholson du Bridge over the River Kwai (David Lean, 1957), dans une double inversion (nationale et sexuelle) de Mme Butterfly (c'est Yonoi qui est attiré par le blanc occidentalisme de Celliers) ${ }^{30}$; même à la fin des années 1990, Bowie se retrouve hôte de la saison 2 de la série érotico-fantastique The Hunger (Showtime, 1997-2000). Les films plus tardifs puisent dans la persona plus « authentique » de Bowie : dans The Prestige et Bandslam, il 
est le mentor (Figure 6) ; dans Mr. Rice's Secret, celui qui apprend à Owen, un adolescent malade du cancer, à profiter de la vie ; et il ouvre chaque épisode de The Hunger en déclamant des généralités métaphysiques souvent à la limite du ridicule.

Fig. 6

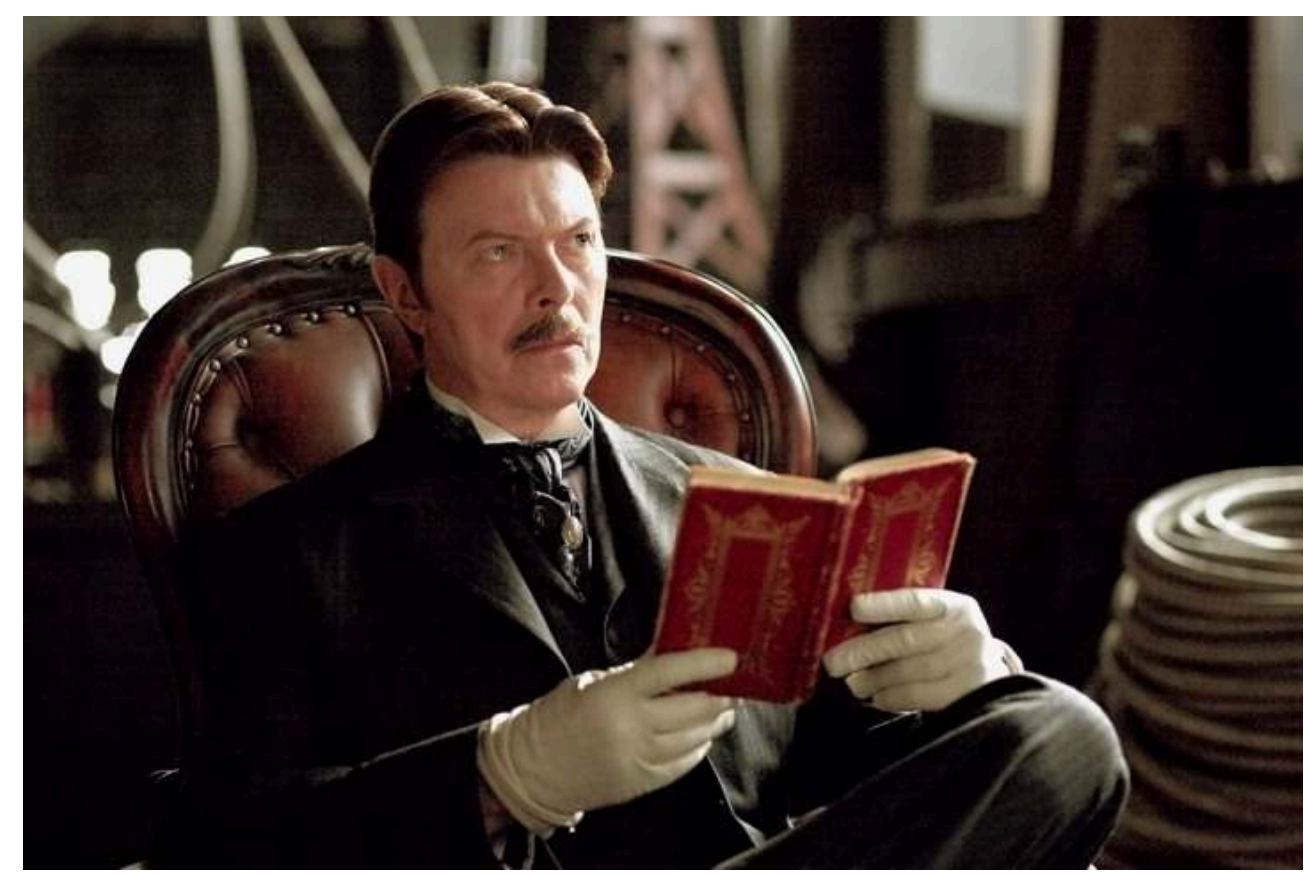

Tesla, l'inventeur d'une machine à téléportation, dans The Prestige (Christopher Nolan, 2006).

Bowie est aussi, comme nous l'avons souligné, une voix. Plusieurs films (Baal, Labyrinth, Absolute Beginners) font appel à son talent vocal dans des numéros, Baal le poète s'adressant directement au spectateur en tant que narrateur chantant (Figure 7). Dans Merry Christmas, Mister Lawrence, Celliers, qui prétend ne pas savoir chanter contrairement à son frère, est néanmoins la voix que Lawrence entend à travers sa cellule, avant de devenir la voix off dans le flash-back qui révèle son sentiment obsédant de culpabilité vis-à-vis de la mort de son frère. Dans Mr. Rice's Secret, les paroles de Mr. Rice accompagnent Owen par-delà la mort. Et dans le générique hypnotique de Lost Highway (David Lynch, 1997), c'est la voix de Bowie qui, avec la chanson «I'm Deranged» (1995), insuffle vie à la route qui défile dans la nuit noire, et par métonymie au film lui-même, évoquant une subjectivité "dérangée » dont "les secrets voyagent", culpabilité qui pourrait bien être celle du protagoniste Fred Madison. Dans l'avant-dernier épisode de Twin Peaks: The Return (Showtime, 2017), le personnage de Phillip Jeffries fait retour malgré la mort de Bowie, ressuscité d'abord par les images recyclées de Twin Peaks: Fire Walk With Me, puis sous la forme d'une théière qui parle, le cliché de la britannicité virant à l'absurde. Bowie, nous dit Lynch, est présent malgré son absence, comme toute star finalement: il rayonne telle une étoile noire à travers tout le champ audiovisuel comme une force culturelle, iconographique et musicale. 
Fig. 7

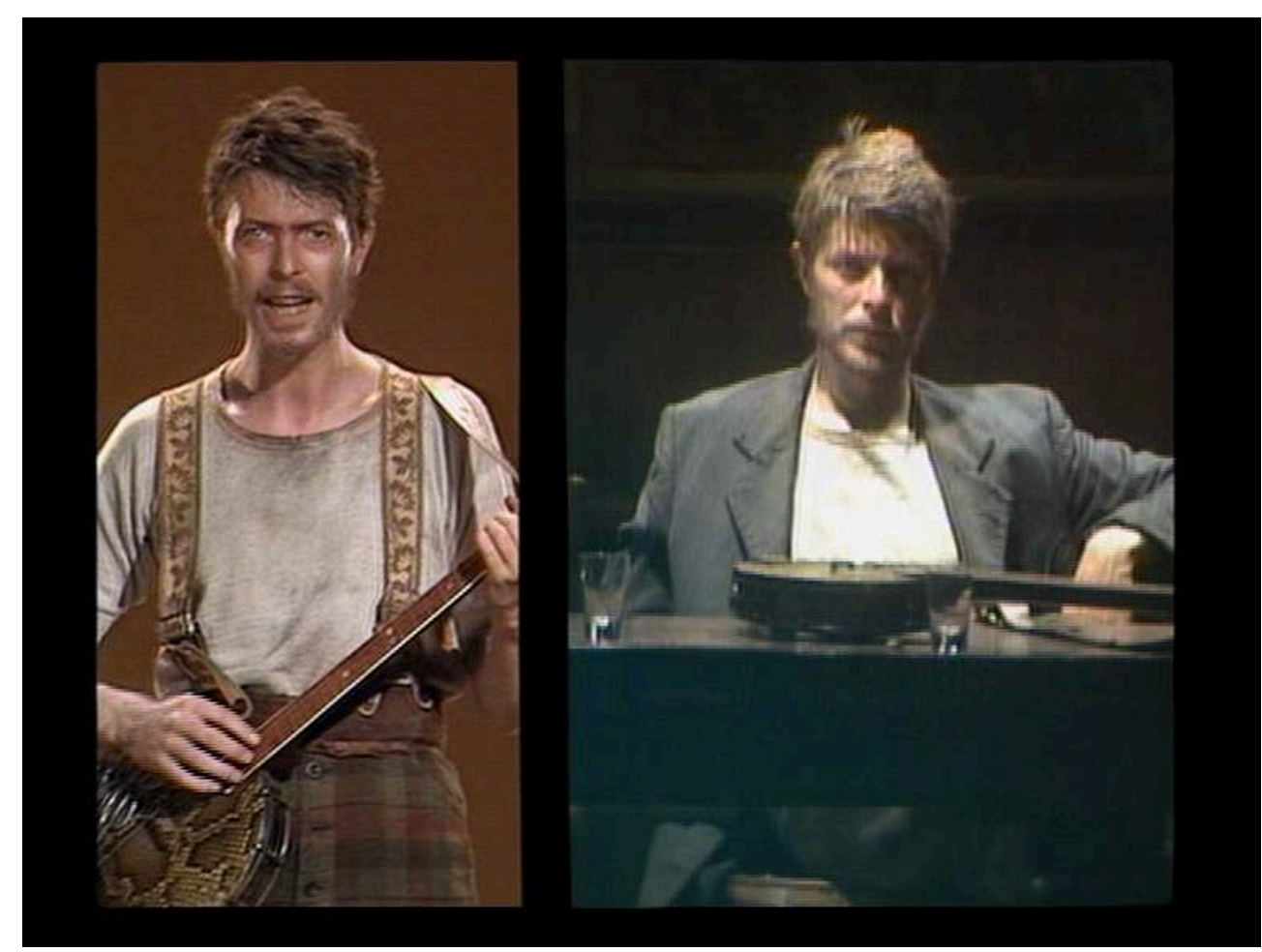

Bowie dans Baal (Alan Clarke, 1982), adaptation télévisuelle d'une pièce de Brecht. dans toutes ses manifestations artistiques, littéraires, sociologiques, historiques, entendues comme autant de signes d'une œuvre ouverte aux approches disciplinaires les plus variées (esthétique, musicologie, gender et queer studies, études culturelles, philosophie, sémiotique, etc.) ; une œuvre dont l'identité, comme celle de son auteur, se caractérise par sa fluidité, sa mobilité, sa quête perpétuelle. Cette carte d'identité est aussi, croyons-nous, celle des auteurs des présentes contributions, que cette œuvre a pu fasciner par la liberté qu'elle cultive et qui se conjugue au talent et à la maîtrise certaine d'un art multiforme.

de ces contributions concernent la réception de Bowie. On sait l'importance que l'étude de la réception a prise en sociologie de la musique et en études de cultures aujourd'hui. Comme l'indique Lawrence Grossberg (38-40), la distinction entre émetteur et récepteur prescrite par le schéma de la communication préserve l'intégrité de la figure de la création, séparant celle-ci de ses appropriations par le public et cantonnant ce dernier à un rôle passif. Elle ne permet donc pas de rendre compte des phénomènes de co-construction des produits culturels populaires où l'engouement et l'implication des fans affectent les contenus dont ils s'emparent. Experiencing David Bowie de Ian Chapman (2015), de même que Bowie de Simon Critchley (2014), donnent l'exemple de telles élaborations, faites d'identification, de fascination, de perplexité, d'ouverture interprétative, lesquelles fonctionnent comme autant d'invitation à se construire avec les fictions visuelles et musicales de Bowie.

Si Claude Chastagner et Shannon Finck abordent cette question, ils suggèrent en revanche que les lectures particulières peuvent tout aussi bien figer la dynamique des propositions de l'artiste : mus par la volonté de les identifier et d'en circonscrire la 
portée pour mieux les saisir, ces destinataires en réduisent la polysémie. Mais la contradiction entre une co-création ouverte et une option interprétative close n'est qu'apparente, toute lecture tendant à réduire la polysémie et à refermer l'horizon du sens.

Shannon Finck examine ainsi le traitement que la critique britannique et américaine fait des albums de Bowie dans les années 1970 pour montrer comment celle-ci résiste aux réinventions de soi que l'artiste leur impose en tentant de lui assigner une identité stable. Elle rappelle que, selon Judith Butler, l'identité se définit par une suite d'actes performatifs. Or, l'investissement personnel des auditeurs dans l'une ou l'autre des incarnations de Bowie, et le rôle dont ils le créditent dans le processus individuel de construction de leur identité les a amenés à se sentir trahis par l'accumulation de ses personnages. Ces changements incessants furent donc perçus comme manque d'authenticité - le sous-entendu étant que Bowie devait se conformer aux attentes de son public. Pourtant, comme dans le striptease étudié par Roland Barthes, l'artiste qui s'expose sous différents atours reste seul maître du jeu, et sa mise en scène de personae transitoires ne fait que jouer avec la possibilité de la nudité comme révélation d'un être véritable.

Pour sa part, Claude Chastagner observe les réponses critiques réservées à Black Star dans la presse anglophone et francophone. Il constate que les commentaires publiés avant la nouvelle du décès de Bowie diffèrent de ceux rédigés postérieurement, les premiers ne relevant pas l'aspect mortifère de l'album cependant que les seconds en font grand cas et le traitent dès lors comme épitaphe. Chastagner reconnaît ainsi dans le ton et la teneur des chroniques françaises les caractéristiques de l'oraison funèbre. À l'inverse, ces traits sont absents de la critique anglophone. S'y substitue le mode de la consolation. Mais l'une et l'autre sont des conventions culturelles établies de longue date qui jurent avec l'esprit innovateur de l'artiste.

On connaît de celui-ci l'usage qu'il a fait du cover (Pin Ups) et sa réexploitation systématique de matériaux antérieurs (ainsi «China Girl» et "Tonight » écrits pour Iggy Pop, «Scream Like a Baby » et « Somebody Up There Likes Me » originellement composés pour Ava Cherry). L'ardeur avec laquelle Bowie s'est battu pour récupérer la propriété de ses premiers albums dont les droits étaient détenus par MainMan suggère un attachement à des phases de créations qu'il avait pourtant mises à distance. Cependant, il se montrait réticent à l'idée de reproduire ses succès antérieurs sur scène et en disque : ainsi lorsqu'il contacta Rykodisk pour qu'ils entreprennent une réédition de tous ses albums, il se mît en quête d'inédits afin d'enrichir chaque $C D$ de quatre à cinq ajouts qui les différencieraient de l'édition précédente (Hallo Spaceboy 44).

Parfois rabaissé au statut d'un compilateur de tendances et d'influences, Bowie intrigue et problématise la notion d'auteur, de sorte qu'il est difficile d'estimer la part d'imitation qui nourrit son œuvre. Il adoptait volontiers le style vocal de Scott Walker, de Bob Dylan, de Lou Reed ou de Bruce Springsteen. La question de l'auteur est également posée par l'implication de multiples collaborateurs dans ses projets musicaux. Mark Ronson fut ainsi mis à contribution pour les arrangements, de même que Toni Visconti, Brian Eno et Robert Fripp, ce dernier insistant sur l'apport unique et transfigurateur de ses guitares sur « Fashion » (voir le reportage de Francis Whately, David Bowie : Five Years [BBC, 2013]).

21 Toutes ces incertitudes donnent lieu dans la critique à une hésitation morale : Bowie doit-il être conçu comme un égoïste exclusivement préoccupé par ses propres intérêts 
ou comme un musicien passionné soucieux de ceux de ses collaborateurs ? Ses avocats, dont Paul Trynka, défendent l'idée que l'artiste n'a rien volé à ses pairs mais qu'il les a inspirés, recueillant le meilleur de ce qui sans son concours n'aurait pu s'exprimer. Si cette question échappe à l'ordre de nos préoccupations, il reste que Bowie fait grand usage de la citation et se nourrit des influences culturelles qui l'environnent.

Grégoire Tosser s'engage dans une étude de la construction harmonique de «Life On Mars? », en soulignant l'ambivalence du rapport à l'original (« Comme d'habitude » de 1967) dont le morceau devait être l'adaptation. Il fait apparaitre une combinaison de ressemblances (mêmes enchaînements d'accords dans le couplet) et de différences par lesquelles le précédent est délibérément mis à distance. Tosser replace le morceau dans le contexte de la production de l'époque, en signalant notamment ses liens avec les usages du glam rock. Il nous invite ainsi à une réflexion sur l'original et la reprise chez Bowie.

Hugues Seress s'intéresse aux albums dits de la "période berlinoise » en s'attachant à les contextualiser dans le lieu et le moment de leur création (Berlin, la seconde moitié des années 1980). Il propose un parallèle entre cette situation de composition et celle de Vienne à la fin du dix-neuvième siècle afin de dégager des conditions déterminantes qui affectent le caractère de Low, Heroes et Lodger. Ce serait une semblable perte de foi, jadis causée par la faillite de l'idéal romantique, qui se répéterait à la fin des années 1970 avec le punk et la cold wave. Partant de cette hypothèse, Seress combine des éléments de musicologie avec sa prise en compte de la spécificité culturelle et historique de la ville.

Un des aspects majeurs du travail de Bowie est bien sûr son caractère spectaculaire. Il ne s'agit pas simplement là d'une affaire de construction de son image publique mais d'une partie constitutive de ses personnages fictionnels, de la manière dont le caractère visuel accuse l'intensité de leur présence, de sorte qu'ils se présentent comme propositions de vie. L'image ainsi élaborée ne vient pas à l'appui d'une carrière musicale mais elle constitue l'une des pièces maîtresses d'une œuvre totale. Cependant elle porte à la visibilité quelque chose d'autre qu'une présence pleine et affirmée : ce qui se révèle est au contraire une figure de l'absence, du détachement, de l'aliénation.

C'est cette figure partielle ou diminuée que Robin Cauche relève dans son analyse du clip de Bowie et Oursler « Where Are We Now? ». Celui-ci reprend les conventions de la lyric video et met ainsi en avant un contenu textuel. objet hybride combinant lyric video et clip promotionnel, «Where Are We Now ? » donne à voir un « corps partiel » puisque seul le visage de l'artiste y apparaît, projeté sur la tête d'une marionnette. De cette représentation résulte un «cache-cache figural » qui contribue, avec la mise du texte au premier plan, à l'effacement du chanteur. (Cet effacement par brouillage et multiplication des signes identitaires, générateurs de confusion, est aussi le sujet de la contribution de Jake Cowan.)

Jonathan Broda et Thomas Schmitt se penchent également sur la visibilité de Bowie, un " artiste fait œuvre », et sur les représentations fictionnelles de lui-même sous les traits de personnages de scène. Ils considèrent la contribution du chanteur-musicien à l'établissement $d u$ standard du clip vidéo par emprunt à des formes médiatiques préexistantes et procèdent à l'étude rapprochée de quatre courts-métrages promotionnels réalisés par Bowie et Mick Rock («Life On Mars? », "John, I’m Only Dancing ", "The Jean Genie ", « Space Oddity ") afin de mettre en lumière ces recours à des dispositifs empruntant à « des contextes déjà médiatisés ». 
27 Jake Cowan témoigne de la constante préoccupation de Bowie par la mortalité tout au long de sa carrière. Le sacrifice répété de ses personae antérieures a fait de lui un artiste perpétuellement renaissant, donc mourant. Il reste engagé dans l'exploration de l'altérité, laquelle ne peut être pensée et définie dans le langage, et il entretient un rapport à la mort comme autre sans lequel le soi ne peut s'affirmer: si la mort est toujours à venir, elle est aussi déjà là avant l'avènement du sujet. Cowan aborde donc Black Star comme nécrologie et se concentre plus spécifiquement sur ce qui, dans l'album, procède à la déconstruction de l'opposition entre vie et mort, donnant à voir et à entendre la porosité et le chevauchement des deux notions. De l'indécision de la formule « David Bowie Is », titre de l'exposition de 2013 au Victoria and Albert Museum, il tire la proposition que Bowie, tout au long de sa carrière, s'est efforcé de devenir un rien (on relira avec profit le volume de Critchley sur ce sujet). En somme, Black Star dévoile le caractère fantomatique de la vie, et entretient un dialogue avec nous par delà la mort.

Enfin, Jean du Verger souligne l'importance du paysage urbain à travers la production de Bowie des années 1960 à 2000 et fait apparaître la cohérence de cette thématique récurrente. Il observe le caractère postmoderne de sa vision de l'environnement. En résultent des visions dystopiques qui traduisent « la nature fragmentée des impressions de l'Amérique de Bowie ». Celles-ci sont inséparables de l'état d'esprit d'un observateur extérieur et distancé qui scrute la ville par la fenêtre. Nous retrouvons là cet œil scrutateur de l'étranger qui ne prend pas part aux activités communes, une présence qui veille.

La somme de ces contributions qui se partage entre musicologie, cultural studies, essais interprétatifs et critique théorique répond, dans sa diversité, à la multiplicité des propositions de Bowie. Elle ne saurait bien sûr en épuiser la fertilité. Mais elle rend compte d'une persistance, d'un esprit qui anime les formes et les genres auxquels l'artiste s'est risqué. Cette présence incertaine, génératrice d'interrogations, n'est pas circonscrite à des limites générationnelles.

Bien sûr, si ce recueil d'articles s'est imposé, c'est que l'occasion d'une célébration de Bowie s'est présentée, et qu'avec celle-ci les conditions étaient réunies qui permettaient de projeter un regard rétrospectif sur sa carrière. Dans de telles circonstances, la tentation était grande de poser un constat sur une œuvre aujourd'hui achevée. Cette sélection se veut ouverte cependant, et sans céder à la manie commémorative ou au réflexe consolateur qui constitueraient, selon claude Chastagner, "un enterrement en grande pompe ", elle exprime la dissémination de traces artistiques bien vivantes, l'épanouissement d'une existence qui s'est diffusée en poussière d'étoile scintillante et dont la constellation éclaire et stimule les arts contemporains, les incitant à se faire mouvement perpétuel, ailes d'un désir inextinguible. Le célèbre cri de Pina Bausch, «Dansez, dansez, sinon nous sommes perdus " (immortalisé en frontispice du documentaire que Wim Wenders lui consacra en 2011), semble faire écho à un autre appel, plus discret car perdu dans les phrasés chaloupés de ce qui, pour être un succès commercial et avoir fait, à ce titre, couler beaucoup d'encre, n'en demeure pas moins un manifeste et, sans doute, un testament artistique et existentiel : «Let's dance / For fear your grace should fall ». Répondre à cet appel, c'est endosser les habits nécessaires à la découverte et à l'accueil de l'étranger, à la jouissance de ces regards démultipliés sur l'autre et sur nous-mêmes auxquels l'art nous convie. 


\section{BIBLIOGRAPHIE}

Arnold, Gina, Daniel Cookney, Kirsty Fairclough and Michael Goddard. Music/Video: Histories, Aesthetics, Media. Londres, New York, Sydney and Delhi: Bloomsbury, 2017.

Broackes, Victoria and Geoffrey Marsh, (ed.). David Bowie Is. Londres: V \& A Publishing, 2013.

Brooker, Will. Forever Stardust: David Bowie Across the Universe. Londres et New York: IB Tauris, 2017.

Buddenberg, Susanne and Thomas Henseler. Berlin: A City Divided. Chronicles. Berlin: avant-verlag, 2012.

Butler, Judith. Gender Trouble: Feminism and the Subversion of Identity, New York and Londres: Routledge, 1990.

Chapman, Ian. Experiencing David Bowie. Londres: Rowman and Littlefield, 2015.

Critchley, Simon. Bowie. n. p.: OR Books, 2014.

Cinque, Toija, Angela Ndalianis and Sean Redmond, (ed.). « In Focus: David Bowie On-Screen. » Cinema Journal 57: 3 (2018): 126-74.

David, Sonia. David Bowie n'est pas mort. Paris : Robert Laffont, 2017.

Deleuze, Gilles. Cinéma 2 : L'Image-temps. Paris : Minuit, 1985.

Devereux, Eoin, Aileen Dillane and Martin J. Power, (ed.). David Bowie: Critical Perspectives. New York and Londres: Routledge, 2015.

Dyer, Richard. Stars. Londres: BFI, 1998 [1979].

Grossberg, Lawrence. We Gotta Get Out of This Place: Popular Conservatism and Postmodern Culture. New York : Routledge, 1992.

Guenassia, Jean-Michel. De l'influence de David Bowie sur la destinée des jeunes filles. Paris: Albin Michel, 2017.

O'Leary, Chris. « Pushing Ahead of the Dame. » <https://bowiesongs.wordpress.com/> (consulté le $08 / 08 / 18$ )

Macé, Marielle. Styles : critique de nos formes de vie. Paris: Gallimard, 2016.

Stevenson, Nick. David Bowie: Fame, Sound and Vision. Cambridge: Polity Press, 2006.

Thibault, Matthieu. David Bowie : l'avant-garde pop. Marseille : Le mot et le reste, 2013.

Thompson, Dave. Hallo Spaceboy: The Rebirth of David Bowie. Toronto: ECW Press, 2006.

Trynka, Paul. Starman: David Bowie, the Definitive Biography. London : Hachette digital, 2011.

Vernallis, Carol. Experiencing Music Video: Aesthetics and Cultural Context. New York: Columbia University Press, 2004.

Waldrep, Shelton. Aesthetics of Self Invention: Oscar Wilde to David Bowie. Minneapolis: University of Minnesota Press, 2004.

-. Future Nostalgia: Performing David Bowie. Londres, New York, New Delhi and Sydney: Bloomsbury, 2015.

David Bowie: 5 Years. dir. Francis Whately, BBC documentary, 2013. 


\section{NOTES}

1. Voir le chapitre «Turn Myself to Face Me: David Bowie in the 1990s and Discovery of Authentic Self » de Bethany Usher et Stephanie Fremaux dans David Bowie: Critical Perspectives.

2. https:// www.independent.co.uk/arts-entertainment/music/news/david-bowie-plaqueunveiled-in-berlin-a7205426.html (consulté le 16 août 2018). On note aussi, en frontispice de la bande dessinée de Suzanne Buddenberg et Thomas Henseler Berlin: A City Divided. Chronicles (2012), la présence du refrain tronqué de « Heroes » tagué sur le mur de Berlin.

3. Voir le compte-rendu de Paul-Emile Bouyssié dans Miranda 14 (2017) https:// journals.openedition.org/miranda/10229.

4. Voir le chapitre « David Bowie Is » de Kathryn Johnson qui ouvre le collectif David Bowie: Critical Perspectives.

5. Voir le compte-rendu de Daniel Huber paru dans Miranda 14 (2017) <https:// journals.openedition.org/miranda/10457>

6. Un terme qui revient souvent dans les articles de la presse et les postes en ligne. Voir, par exemple, l'article, "Les légendes du rock \#4 : David Bowie, l'homme qui venait d'ailleurs ", de Thomas Malher sur Le Point.fr, publié le 26/12/2013. Vu le 2/7/2018.

7. Dans un article intitulé "How David Bowie Impacted my Sexuality and Gender Fluidity", Kristen Sollee déclare : "Before I read Judith Butler's declaration in Gender Trouble that gender is a social performance and "a fantasy instituted and inscribed on the surface of bodies," I knew it to be true from watching David Bowie ». Voir Bustle.com, 1/11/2016, https://www.bustle.com/ articles/134812-how-david-bowie-impacted-my-sexuality-and-gender-fluidity.

8. Voir Kathryn Johnson.

9. On se souvient de la chanson «Beau oui comme Bowie » (1983) écrite et composée par Serge Gainsbourg et interprétée par Isabelle Adjani.

10. <https://www.youtube.com/watch?v=J2V1CHTWLOU> (consulté le 3 juillet 2018).

11. Voir “Art's Filthy Lesson" de Tiffany Naiman et "Revisiting Bowie's Berlin" de David Buckley dans David Bowie: Critical Perspectives.

12. Voir Kathryn Johnson.

13. https://www.youtube.com/watch?v=D67kmFzSh_o

14. https://www.youtube.com/watch? $\mathrm{v}=\mathrm{kszLwBaC} 4 \mathrm{Sw}$

15. https://www.youtube.com/watch?v=iYYRH4apXDo

16. https://www.youtube.com/watch?v=dOy7vPwEtCw

17. https://www.youtube.com/watch?v=CMThz7eQ6K0

18. Voir l'étude des masques et du maquillage dans les clips « Boys Keep Swinging » (1979), « Blue Jean » (1984) et «Thursday's Child» (1999), de Julie Lobalzo Wright dans Music/Video: History, Aesthetics, Media.

19. https://www.youtube.com/watch?v=v--IqqusnNQ

20. https://www.youtube.com/watch?v=Tgcc5V9Hu3g

21. https://www.youtube.com/watch?v=E_8IXx4tsus

22. https://www.youtube.com/watch?v=N4d7Wp9kKjA

23. Voir "The "China Girl" Problem: Reconsidering David Bowie in the 1980s » dans David Bowie: Critical Perspectives.

24. https://www.youtube.com/watch?v=QWtsV50_-p4

25. Voir «Embodying Stardom, Representing Otherness: David Bowie in Merry Christmas Mr. Lawrence » dans David Bowie: Critical Perspectives

26. Pour une comparaison plus élaborée de Bowie et de Madonna, voir Madonna as Postmodern Myth de Georges-Claude Gilbert (3, 38-39, 111-112).

27. Voir « Moss Garden: David Bowie and Japonism in fashion in the 1970s » d'Helence Marie Thian dans David Bowie: Critical Perspectives. 
28. Richard Dyer a introduit ce concept dans Stars $(60,63)$.

29. Voir le chapitre de Wright dans David Bowie: Critical Perspectives qui analyse principalement The Man Who Fell to Earth, The Hunger et Merry Christmas, Mr. Lawrence, et « David Bowie's Perverse Cinematic Body » de Galt qui analyse The Man Who Fell to Earth, The Hunger et Merry Christmas, Mr. Lawrence et Labyrinth.

30. Voir l'excellente analyse de la scène du baiser dans le chapitre «Embodying Stardom, Representing Otherness: David Bowie in Merry Christmas Mr. Lawrence » de Mehdi Derfoufi dans David Bowie: Critical Perspectives.

\section{RÉSUMÉS}

Le présent article est l'introduction d'un numéro de Miranda consacré aux paysages et aux héritages de David Bowie à la suite d'une journée d'étude organisée en décembre 2016 à l'Université Toulouse-Jean Jaurès. Ouvrant la voie à une série d'études minutieuses des multiples facettes de la carrière de Bowie, des influences qui l'ont nourrie et du pouvoir d'inspiration qu'elle possède à son tour, il se propose de passer en revue et de distinguer les composantes d'un art dont la complexité s'envisage comme un kaléidoscope des rêves, des désarrois, des courants, images et dissonances qui hantent notre époque contemporaine.

The present article is an introduction to an issue of Miranda devoted to David Bowie's landscapes and heritage in the wake of a one-day conference held at the University Toulouse-Jean Jaurès in December 2016. Setting the stage for a succession of close investigations into Bowie's multifaceted career, its influences and its own influential power, it aims to survey and foreground various components of a complex body of work, which represents a kaleidoscope of the dreams and disarrays, of the trends, images and dissonances that keep haunting our contemporary world.

\section{INDEX}

Mots-clés : Bowie, héritage, identité, musique, performance, pop culture, théâtre, cinéma, vidéo Keywords : Bowie, heritage, identity, music, performance, pop culture, drama, cinema, video

\section{AUTEURS}

\section{PHILIPPE BIRGY}

Professeur à l'UT2J

birgy@univ-tlse2.fr

\section{DAVID ROCHE}

Professeur à l'UT2J

mudrocka@gmail.com 


\section{NATHALIE VINCENT-ARNAUD}

Professeur à l'UT2J

nathalie.vincent-arnaud@univ-tlse2.fr 